\title{
Structural Determination of Picomole Amounts of Phospholipids Via Electrospray Ionization Tandem Mass Spectrometry
}

\author{
Xianlin Han and Richard W. Gross \\ Division of Bioorganic Chemistry and Molecular Pharmacology, Departments of Medicine, Chemistry, and \\ Molecular Biology \& Pharmacology, Washington University School of Medicine, St. Louis, Missouri, USA
}

\begin{abstract}
The remarkable sensitivity of electrospray ionization was exploited to achieve great increases in the sensitivity of tandem mass spectrometric analyses of phospholipids derived from both synthetic and biologic sources. Herein, we demonstrate that (1) product-ion spectra after electrospray ionization can be obtained easily by utilizing $\leq 5$ pmol of phospholipid with a mass-selected window of less than 2 mass units, (2) the low energy inherent in the electrospray ionization method facilitates analysis of labile molecular ions that are not easily detected with the relatively high energy employed during fast-atom bombardment desorption, and (3) collision-induced dissociation of precursor ions generated from electrospray ionization often resulted in novel product-ion patterns. Collectively, these results underscore the utility of electrospray ionization tandem mass spectroscopy for the structural determination of diminutive amounts of phospholipids. ( $\mathrm{Am}$ Soc Mass Spectrom 1995, 6, 1202-1210)
\end{abstract}

$\mathrm{R}$ ecently, electrospray ionization mass spectroscopic analyses of phospholipid constituents obtained from synthetic or biologic sources have facilitated quantification of individual molecular ions from subpicomole amounts of phospholipid molecular species [1-4]. However, as previously demonstrated by other soft ionization techniques (e.g., [5-11]), the quantification and structural determination of phospholipid mixtures via the electrospray ionization technique requires further analyses of individual molecular ion peaks by tandem mass spectrometry to discriminate among constituent isobaric molecular species and individual regioisomers. We recognized that the extraordinary sensitivity of the electrospray ionization method could be exploited to facilitate similar dramatic increases in the sensitivity of tandem mass spectroscopic analysis of phospholipids, and thereby provide critical structural information from diminutive amounts of synthetic or biologic material. Furthermore, the low energy ionization inherent in the electrospray ionization technique was anticipated to allow access to new product ions that were not detected easily by using fast-atom bombardment (FAB) ionization. We now report the utilization of electrospray ionization for both the positive- and negative-ion mode tandem mass spectroscopic analysis of picomole amounts of phospholipids consumed and demonstrate the importance of collisional activation of low energy

Address reprint requests and correspondence to Dr. Richard W. Gross, Division of Bioorganic Chemistry and Molecular Pharmacology, Washington University School of Medicine, 660 South Euclid, Box 8020 , St. Louis, MO 63110. quasimolecular ions to generate new product ions useful in the structural determination of individual phospholipid molecular species.

\section{Materials and Methods}

\section{Materials}

All commercially available phospholipids were obtained from Avanti Polar Lipids, Inc. (Alabaster, AL). 1-Octadecanoyl-2-eicosa tetra-5' ${ }^{\prime} 8^{\prime}, 11^{\prime}, 14^{\prime}$-enoyl-snglycero-3-phosphoethanolamine, 1-O-(Z)-octadec-1'enyl-2-eicosatetra-5', ' $^{\prime}, 11^{\prime}, 14^{\prime}$-enoyl-sn-glycero-3-phosphoethanolamine, and other plasmenylethanolamine molecular species were freshly purified from crude bovine heart or bovine brain ethanolamine glycerophospholipids by reverse-phase high-performance liquid chromatography (HPLC) as described previously $[12,13]$. Plasmenylcholine and plasmanylcholine molecular species were synthesized and purified by methods previously described [14]. The mass of synthetic phospholipids employed in this study was quantified by capillary gas chromatography after acid methanolysis by comparisons with internal standard (arachidic acid) [15].

\section{Electrospray Ionization Tandem Mass Spectrometry}

Electrospray ionization (ESI) mass spectrometric analyses were performed via a triple-quadrupole tandem mass spectrometer (Finnigan-MAT TSQ 700, San Jose, CA) equipped with an electrospray ionization interface 
(Analytica of Branford, Branford, CT) as described previously in detail [1]. In brief, both the electrospray needle and the skimmer were operated at ground potential, whereas the electrospray chamber and metalized entrance of the glass capillary were operated at $-3.5 \mathrm{kV}$ in the positive-ion mode and at $+3.0 \mathrm{kV}$ in the negative-ion mode. A positive or negative $90-\mathrm{V}$ potential was applied to the metalized exit of the glass capillary for positive- or negative-ion spectra, respectively. A separate positive or negative $175-\mathrm{V}$ potential was placed on the tube lens for acquisition of positive or negative ions, respectively. Tandem mass spectrometry of phospholipids after electrospray ionization was performed by passage of the mass-selected precursor ion of individual phospholipids (less than 2 mass units in typical experiments) from the first quadrupole into the second quadrupole, where dissociation was induced through collisional activation with argon gas. The mass of the resultant product ions was analyzed after passage into the third quadrupole. The degree of collisional activation was adjusted through variation of the dc offset voltage and collision gas pressures. During this study, collision energies of $18-25 \mathrm{eV}$ (with respect to the laboratory reference) and collision gas pressures of 2.5-3.5 mtorr were used. The third quadrupole was tuned to obtain unit mass resolution for all experiments during this study and the mass of all ions was rounded to the nearest integer. All product-ion spectra were acquired by using a signalaveraging protocol in the profile mode with a scan rate of $200-400 \mathrm{u} / \mathrm{s}$. Typically, a 5-min period of signal-averaging was used for each tandem mass spectrum. Signal-averaging and processing of the spectra were accomplished with a DEC station 3100 equipped with an ULTRIX operating system that used commercial software (ICIS, Finnigan). All samples (0.2-1 $\mathrm{pmol} / \mu \mathrm{L}$ ) were dissolved in 1:2 chloroform-methanol and infused directly into the ESI chamber by using a syringe pump (model 22, Harvard Apparatus, South Natick, MA) at a flow rate of $1 \mu \mathrm{L} / \mathrm{min}$ for $5 \mathrm{~min}$. Excess chloride ( $1 \mu \mathrm{mol} / \mathrm{mL}$ of $\mathrm{NaCl}$ ) was added in the infusion solution for negative-ion electrospray ionization tandem mass spectrometry (ESI-MS/MS) analyses of choline-containing phospholipids.

\section{Results and Discussion}

\section{Tandem Mass Spectrometry of Choline Glycerophospholipids}

The utility of ESI-MS/MS for phospholipid structural determination was achieved through negative-ion tandem mass spectra of the chlorine adduct of choline glycerophospholipids (Figure 1). After collisional activation of the chlorine adduct ion $\left[\mathrm{M}+\mathrm{Cl}^{-}\right.$of choline glycerophospholipids [the major negatively charged quasimolecular ion when excess chloride $(1 \mu \mathrm{mol} / \mathrm{mL})$ was added in the infused solution], multiple product ions were detected; each represented salient structural information. First, an abundant ion $\left([\mathrm{M}-15]^{-}\right.$, which corresponds to $N, N$-dimethylethanolamine glycerophospholipid) that results from loss of $\mathrm{CH}_{3} \mathrm{Cl}$ from
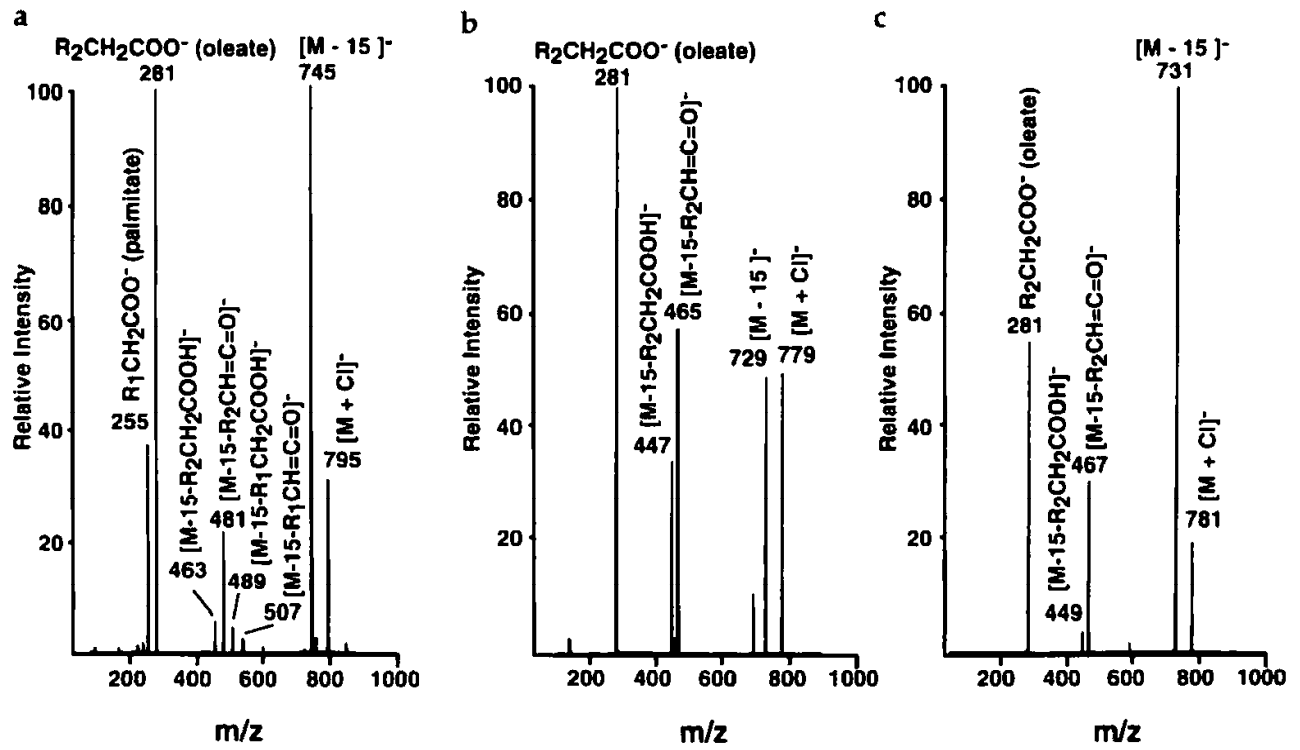

Figure 1. Negative-ion ESI tandem mass spectra of the chlorine adduct of choline glycerophospholipids. (a) ESI-MS/MS of the chlorine adduct of 1-hexadecanoyl-2-octadec-9'-enoyl-sin-glycero-3phosphocholine. (b) ESI-MS/MS of the chlorine adduct of 1-O-(Z)-hexadec-1'-enyl-2-octadec-9'-enoyl-sn-glycero-3-phosphocholine (plasmenylcholine). (c) ESI-MS/MS of chlorine adduct of 1-Ohexadecyl-2-octadec-9'-enoyl-sn-glycero-3-phosphocholine (plasmanylcholine). Each choline glycerophospholipid ( $1 \mathrm{pmol} / \mu \mathrm{L}$ ) was dissolved in 1:2 chloroform-methanol with the addition of 1 $\mu \mathrm{mol} / \mathrm{mL}$ of $\mathrm{NaCl}$. After selection of the quasimolecular ion in the first quadrupole, collision activation was performed in the second quadrupole and the resultant product ions were analyzed in the third quadrupole as described in the Materials and Methods section. 
the chlorine adduct of 1-hexadecanoyl-2-octadec-9'enoyl-sn-glycero-3-phosphocholine was present (Figure 1a). This product ion is diagnostic for the presence of the choline moiety. Second, a cluster of peaks (near $m / z \quad 480$ ) that corresponds to $N, N$-dimethylethanolamine lysoglycerophospholipidlike ions was present. These product ions contain important information about the regiospecificity of the acyl chain linkages in the parent choline glycerophospholipid (Figure 1a). These lysoglycerophospholipidlike product ions and thus, the information contained therein, were difficult to observe via fast-atom bombardment-tandem mass spectrometry (FAB-MS/MS) [16-21] because of the limited quasimolecular ion intensity of choline glycerophospholipids after fast-atom bombardment (FAB) ionization in the negative-ion mode. Third, because the intensity of the product ion that corresponds to the sn-2 acyl substituent is typically threefold greater than that of the carboxylate anion derived from cleavage of the acyl constituent at the $s n-1$ carbon from asymmetrical phosphatidylcholines (16:0-18:1, 16:0-18:2, 18:0-18:1, and 16:0-20:4 molecular species examined) (e.g., Figure 1a), the regiospecificity of the acyl chain distribution for these asymmetrical choline glycerophospholipids can be clarified. Although similar ratios of product-ion peaks have previously been demonstrated utilizing FAB-MS/MS of phosphatidylcholine in the negative-ion mode $[18,20]$, we point out the greatly enhanced sensitivity of ESI-MS/MS facilitates structural determination from $<5$ pmol of phospholipid (consumed).

Negative-ion ESI-MS/MS of the chlorine adduct plasmenylcholine results in the production of product ions whose relative abundances are substantially different from those observed for phosphatidylcholine (Figure 1b). First, the relative abundance of the $\mathrm{N}, \mathrm{N}$-dimethyl plasmenylethanolamine anion (i.e., $[M-15]^{-}$) was much less than that from its diacyl phospholipid counterpart. Second, the peak intensities of the lysophospholipidlike product anions that resulted from the chlorine adduct of plasmenylcholine were greater than those of their monoacyl lysophospholipidlike counterparts that resulted from the corresponding chlorine adduct of phosphatidylcholine. These features likely result from the relatively higher stability and formation rate of the sn-1 vinyl etherlinked product ion in comparison to the counterpart for the loss of the $s n-1$ carboxylic acid (or derivative) under conditions in which the sn-2 acyl group is lost easily. Cleavage of the vinyl ether linkage at the $s n-1$ position of choline plasmalogens does not occur to a significant extent (i.e., product ions that correspond to the anticipated products from vinyl ether cleavage were not detected). Third, only a single carboxylate anion is present in product-ion spectra of the chlorine adduct plasmenylcholine, which reflects loss of the acyl moiety from the sn-2 position and the absence of an acyl group at the $s n-1$ position. ESI-MS/MS of the chlorine adduct plasmanylcholine resulted in a qualita- tively similar product-ion pattern to that manifest by the chlorine adduct plasmenylcholine (Figure $1 \mathrm{~b}$ ), but the product-ion abundance was different (Figure 1c). For instance, the relative abundance of the sn-2 carboxylate anion that resulted from the chlorine adduct of plasmanylcholine was approximately half that of its plasmenylcholine chlorine adduct counterpart. In contrast, the product ion at $[M-15]^{-}$from the chlorine adduct of plasmanylcholine was more abundant than that which resulted from the chlorine adduct of plasmenylcholine. These observations were documented by utilizing molecular species with multiple different chain lengths and double bonds at the $s n-2$ position $(16: 0-16: 0,16: 0-18: 1, \quad 16: 0-20: 4,18: 0-18: 1$, and 18:0-20:4 molecular species were examined).

It previously has been demonstrated that the protonated choline glycerophospholipid ions (i.e., $\left[\mathrm{M}+\mathrm{H}^{+}\right.$ ions) dominate sodiated ions when the $\mathrm{pH}$ of the infused choline glycerophospholipid solutions is decreased [1]. The only positive ion of choline glycerophospholipids available for ESI-MS/MS analysis under the experimental conditions employed is the sodium adduct (i.e., $[\mathrm{M}+\mathrm{Na}]^{+}$) [1]. Positive-ion ESIMS/MS of sodiated choline glycerophospholipids (i.e., $[\mathrm{M}+\mathrm{Na}]^{+}$ions) yielded dramatically different collision-induced dissociation patterns from the protonated choline glycerophospholipids. Product-ion spectra of all sodiated choline glycerophospholipids acquired by using ESI-MS/MS displayed four abundant product ions. As examples, the positive-ion ESIMS/MS spectra of each choline glycerophospholipid subclass from 5 pmol of material (consumed) are shown in Figure 2. Each of these product ions corresponds to either the loss of trimethylamine $\left([\mathrm{M}+\mathrm{Na}-59]^{+}\right)$, the production of diglyceridelike cations (i.e., $[\mathrm{M}+\mathrm{Na}$ $-183]^{+}$and $[\mathrm{M}+\mathrm{Na}-205]^{+}$), or the generation of a sodiated five-member cyclophosphane $(\mathrm{m} / \mathrm{z}$ 147) (Figure 2). Some of these product ions were similar or identical to the fragment ions of diacyl phosphatidylcholine acquired by using thermospray mass spectrometry and were identified previously [22]. This collision-induced dissociation pattern after electrospray ionization is consistent with the possible pathways proposed in Scheme I. The presence of both pathway 3 and pathway 4 in the proposed scheme has been verified by employing sn-2 monoacyl lysophosphatidylcholine and $s n-1$ monoacyl lysophosphatidylcholine, respectively (data not shown). In contrast to the collision-induced dissociation pattern observed from sodium adducts after ESI, collisional activation of the protonated choline glycerophospholipids after electrospray ionization (spectra not shown) and FAB ionization $[5,21,23]$ resulted in only a single product ion (i.e., phosphocholine cation, $m / z$ 184). The positive-ion ESI-MS/MS spectra of sodiated phosphatidylcholines can be further distinguished from their sodiated plasmenylcholine and sodiated plasmanylcholine counterparts by the presence of a relatively abundant $[M+$ $\mathrm{Na}-183]^{+}$product ion as observed from the analyses 
a

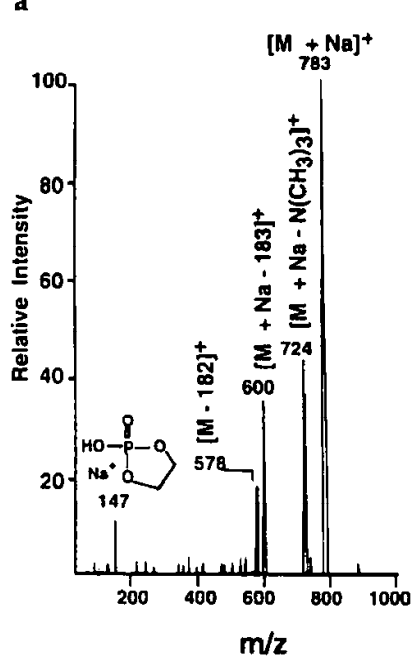

b

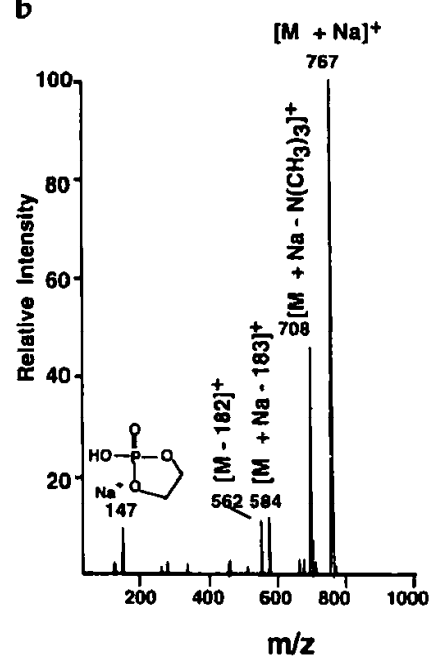

c

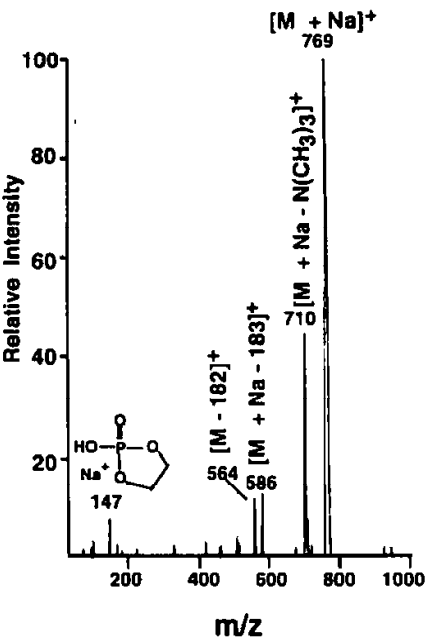

Figure 2. Positive-ion ESI tandem mass spectra of sodiated choline glycerophospholipids. (a) ESI-MS/MS of sodiated 1-hexadecanoyl-2-octadec-9'-enoyl-sn-glycero-3-phosphocholine. (b) ESIMS/MS of sodiated 1-O-(Z)-hexadec-1'-enyl-2-octadec-9'-enoyl-sn-glycero-3-phosphocholine. (c) ESI-MS/MS of sodiated 1-O-hexadecyl-2-octadec-9'-enoyl-sll-glycero-3-phosphocholine. Each choline glycerophospholipid (1 pmol $/ \mu \mathrm{L}$ ) was dissolved in 1:2 chloroform-methanol prior to performance of ESI tandem mass spectrometry as described in the Materials and Methods section.

of 16:0-16:0, 16:0-18:1, 16:0-20:4, and 18:0-20:4 molecular species in each subclass.

\section{Tandem Mass Spectrometry of Sphingomyelins}

The positive-ion ESI mass spectra of commercially available bovine brain sphingomyelin $(1 \mu \mathrm{g} / \mathrm{mL}$ in $1: 2$ chloroform-methanol) displayed six sodiated sphingomyelin ion peaks at $m / z 726,754,782,810,836$, and 838 (Figure 3a), which correspond to the following $N$-acyl amides: C16:0, C18:0, C20:0, C22:0, C24:1, and $\mathrm{C} 24: 0$. The peak intensities of these ions are in excel-

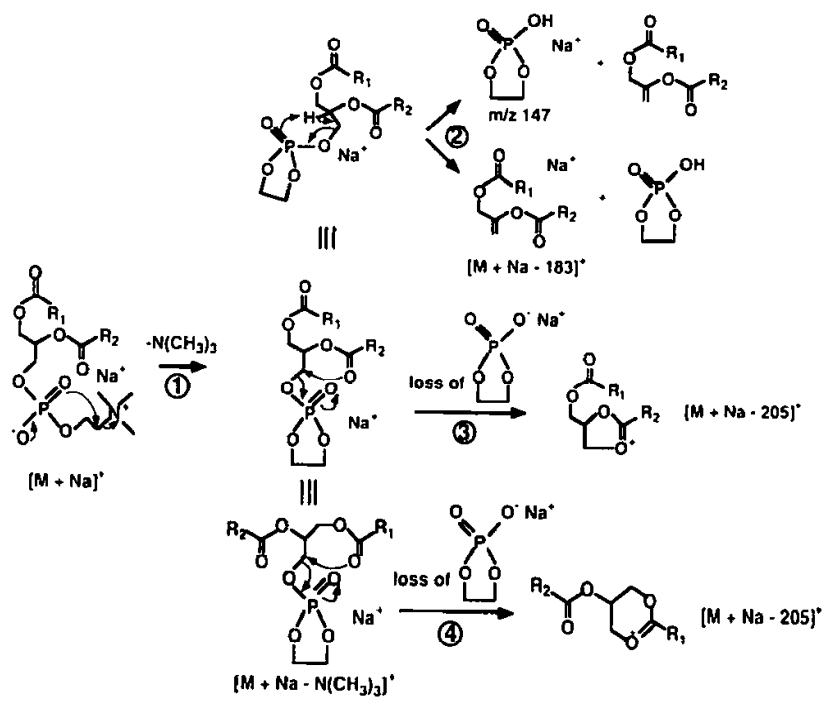

Scheme I. Proposed collision-induced dissociation pathways of sodiated choline glycerophospholipids after electrospray ionization. Pathway 4 is not present in the collisional activation of both plasmenylcholines and plasmanylcholines. lent agreement with the reported molecular species distribution present in sphingomyelins derived from bovine brain (data sheet provided by Avanti Polar Lipids, Inc., Alabaster, AL).

The presence of a phosphocholine polar head group in sphingomyelins renders positive-ion ESI-MS/MS spectra of sodiated sphingomyelins similar to those of sodiated choline glycerophospholipids (compare Figure $3 \mathrm{~b}$ to Figure 2). Selection and collisional activation of the ion at $m / z 754$ (i.e., sodiated $d 18: 1-18: 0$ sphingomyelin) after ESI yields several informative product ions (Figure $3 \mathrm{~b}$ ). The most abundant product ion is $m / z$ 695, which corresponds to the loss of trimethylamine $\left([\mathrm{M}+\mathrm{Na}-59]^{+}\right)$. Other product ions at $\mathrm{m} / \mathrm{z}$ 571,549 , and 531 correspond to three ceramidelike cations (i.e., $[\mathrm{M}+\mathrm{Na}-183]^{+},[\mathrm{M}+\mathrm{Na}-205]^{+}$, and $\left[\mathrm{M}+\mathrm{Na}-205-\mathrm{H}_{2} \mathrm{O}\right]$, respectively). The product ion at $m / z 147$ corresponds to the sodiated five-member cyclophosphane. A distinguishing product ion in ESIMS/MS spectra of sodiated sphingomyelins as compared to those of sodiated choline glycerophospholipids is the presence of a $\left[\mathrm{M}+\mathrm{Na}-205-\mathrm{H}_{2} \mathrm{O}\right]^{+}$) ion due to the facile loss of $\mathrm{H}_{2} \mathrm{O}$ from the sphingosine.

Negative-ion ESI mass spectra of sphingomyelin displayed a predominant chlorine adduct ion (i.e., $\left[\mathrm{M}+\mathrm{Cl}^{-}\right)$when spectra were obtained in the presence of chloride ion. Selection and collisional activation of the chlorine adduct ion of sphingomyelin after ESI yields one major product ion (i.e., $[M-15]^{-}$) that corresponds to the loss of $\mathrm{CH}_{3} \mathrm{Cl}$ (Figure 3c). Therefore, negative-ion ESI-MS/MS spectra of the chlorine adduct of sphingomyelins, in contrast to positive-ion ESI-MS/MS spectra of sodiated sphingomyelins, do not provide additional structural information. This observation suggests that there is insufficient accessible 

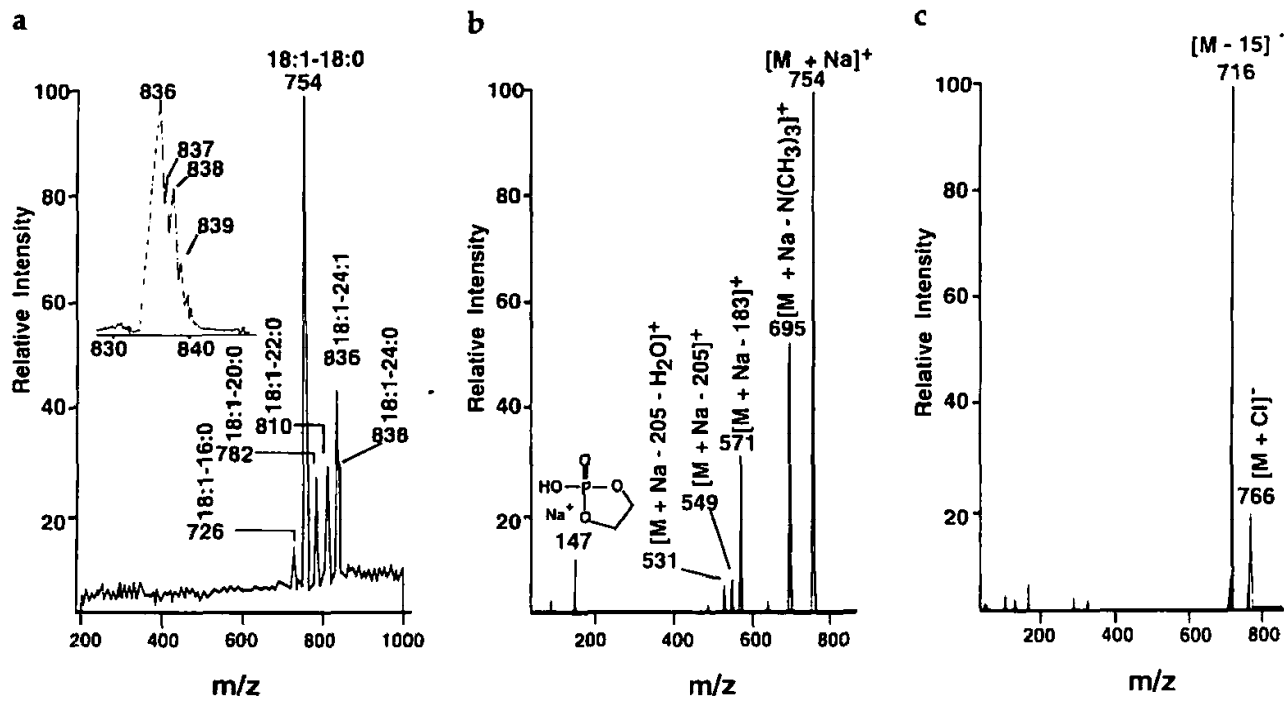

Figure 3. ESI mass spectrometry of sphingomyelins. (a) Positive-ion ESI mass spectrum of brain sphingomyelins. Six sodiated sphingomyelin ions were detected and defined by the length of their ceramide carbon number and $N$-acyl linkage (e.g., d18:1-18:0 sphingomyelin corresponds to $\mathrm{N}$-stearoyl-trans-4-sphingenine-1-phosphorylcholine.). (b) Positive-ion ESI tandem mass spectrum of selected sodium adducts of sphingomyelin. (c) Negative-ion ESI tandem mass spectrum of selected chlorine adduct of sphingomyelin. Sphingomyelins $(5 \mathrm{pmol} / \mu \mathrm{L})$ was dissolved in $1: 2$ chloroform-methanol and negative-ion ESI mass spectrometry of brain sphingomyelin was performed in the presence of $\mathrm{NaCl}$ in the sample solution that demonstrates six chlorine adduct ions.

collision energy in the quadrupole system to dissociate the acyl amide derivatives due to the relatively high stability of the amide linkage.

\section{Tandem Mass Spectrometry of Ethanolamine Glycerophospholipids}

The most abundant product ions in negative-ion ESIMS/MS of deprotonated ethanolamine glycerophospholipids are the carboxylate anion(s) (Figure 4), which can be used to identify the regiochemistry of asymmetrical phosphatidylethanolamines as seen previously via FAB-MS/MS $[6,21,24]$. The ratio of product ions that result from the $s n-2$ carboxylate anion to those that result from the $s n-1$ carboxylate ion is approximately 3:1 for chain lengths between 16 and 20 carbons that contain up to four double bonds (Table 1). For molecular species that contain 22 carbons and more than 4 double bonds, an approximate value of 1 for this ratio was obtained (Table 1). Thus, the regiospecificity of the acyl moieties in asymmetrical phosphatidylethanol-
Figure 4. Negative-ion ESI tandem mass spectra of deprotonated ethanolamine glycerophospholipids. (a) ESI-MS/MS of deprotonated 1-octadecanoyl-2-eicosatetra-5', 8', 11', 14'-enoyl-sn-glycero-3phosphoethanolamine. (b) ESI-MS/MS of deprotonated 1-O-(Z)-octadec-1'-enyl-2eicosatetra-5', 8', 11', 14'-enoyl-sn-glycero-3-phosphoethanolamine (plasmenylethanolamine). Each ethanolamine glycerophospholipid $(0.2 \mathrm{pmol} / \mu \mathrm{L})$ was dissolved in 1:2 chloroform-methanol prior to performance of ESI product-ion analysis as described in the Materials and Methods section.
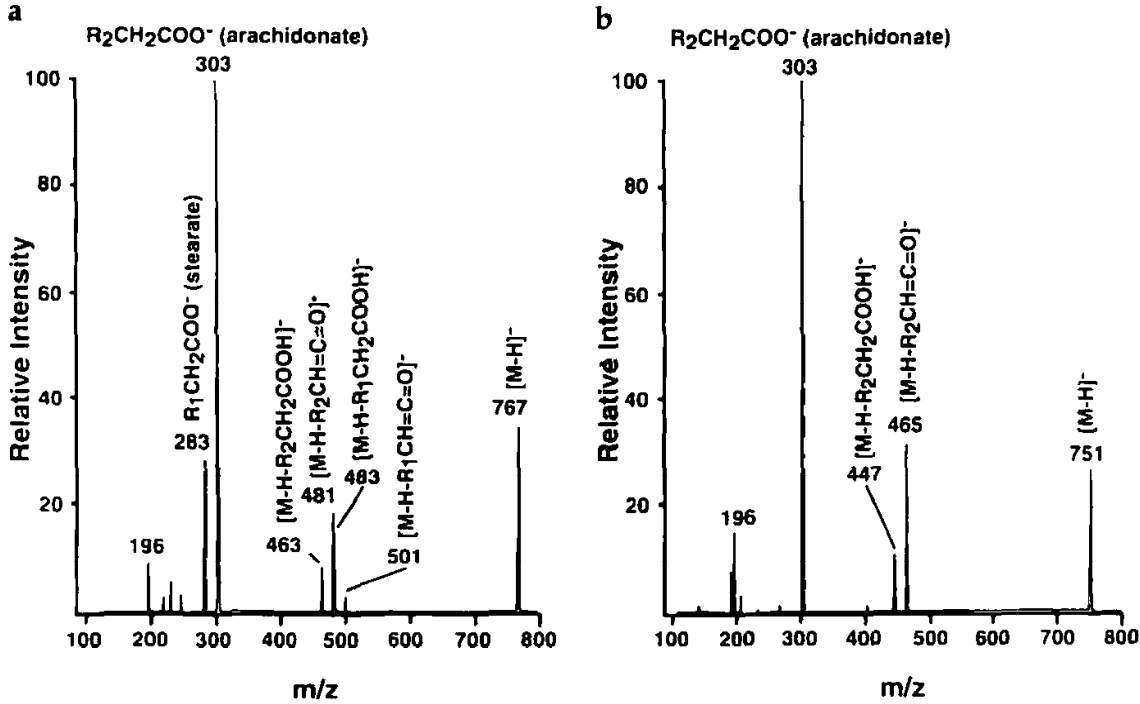
Table 1. The relative intensity ratios of $s n-2$ carboxylate anion $\left(\mathrm{R}_{2} \mathrm{COO}^{-}\right)$versus $\mathrm{sn}-1$ carboxylate anion $\left(\mathrm{R}_{1} \mathrm{COO}^{-}\right)$ following collision-induced dissociation of deprotonated asymmetric phosphatidylethanolamine molecular species $\left([\mathrm{M}-\mathrm{H}]^{-}\right)$after $\mathrm{ESI}^{\mathrm{a}}$

\begin{tabular}{lll}
\hline$[\mathrm{M}-\mathrm{H}]^{-}$ & $\mathrm{MW}$ & $\mathrm{R}_{2} \mathrm{COO}^{-} / \mathrm{R}_{1} \mathrm{COO}^{-}$ \\
\hline \hline $16: 0-18: 1$ & 718 & $3.08 \pm 0.35^{\mathrm{b}}$ \\
$16: 0-18: 2$ & 716 & 2.86 \\
$16: 0-20: 4$ & 740 & 2.93 \\
$16: 0-22: 4$ & 768 & 1.42 \\
$16: 0-22: 5$ & 766 & 1.11 \\
$16: 0-22: 6$ & 764 & 1.05 \\
$18: 0-18: 1$ & 746 & 2.84 \\
$18: 0-18: 2$ & 744 & 2.88 \\
$18: 0-20: 4$ & 768 & $3.11 \pm 0.37^{\mathrm{b}}$ \\
$18: 0-22: 4$ & 780 & 1.29 \\
$18: 0-22: 5$ & 794 & 1.25 \\
$18: 0-22: 6$ & 792 & 0.92 \\
$18: 1-20: 4$ & 766 & 2.76 \\
$18: 1-22: 4$ & 794 & 1.33 \\
$20: 0-20: 4$ & 796 & 2.34 \\
\hline
\end{tabular}

\footnotetext{
Individual molecular species (16:0-18:1, 16:0-18:2, 16:0-20:4, 18:0-18:1, and 18:0-18:2) were obtained from Avanti Polar Lipids, Inc. Other molecular species were purified from natural sources by reverse-phase HPLC purification as described in the Materials and Methods section. Fatty acyl groups were designated by the convention carbon number:double bond number.

The standard deviation was obtained from the analyses of the species that were derived from multiple sources.
}

amine can be assigned directly. In addition, the regiospecificity of the acyl moieties in asymmetrical phosphatidylethanolamine also can be confirmed from the packet of $s n-2$ and $s n-1$ acyl lysophosphatidylethanolaminelike product-ion peaks that correspond to the neutral loss of the alkyl ketene and/or the carboxylic acid from the $s n-1$ and $s n-2$ positions because the $s n-1$ acyl lysophosphatidylethanolaminelike anion is always more abundant than the $s n-2$ acyl lysophosphatidylethanolaminelike ion (Figure 4a). The negative-ion ESI-MS/MS spectra of deprotonated phosphatidylethanolamine and plasmenylethanolamine contain diagnostic peaks at $m / z 196$, which correspond to the loss of $\mathrm{H}_{2} \mathrm{O}$ from glycerol phosphoethanolamines (Figure 4). This peak was not identified previously in FAB-MS/MS studies of ethanolamine glycerophospholipids. In the negative-ion ESI-MS/MS of deprotonated plasmenylethanolamine, the presence of a relatively more intense lysoplasmenylethanolaminelike ion peak relative to its phosphatidylethanolamine counterpart from examined plasmenylethanolamine molecular species that contain 16:0, 18:0, 18:1, $18: 2,20: 1$, and $20: 4$ acyl chains at $s n-2$ position indicates the presence of plasmenylethanolamine (Figure 4). This product ion ratio likely results from the relatively higher stability and formation rate of the $s n-1$ vinyl ether-linked product ion from plasmenylethanolamine anion in comparison to those that result from the loss of the sn-1 carboxylic acid (or derivative) from deprotonated phosphatidylethanolamine (similar to the case of plasmenylcholine versus phosphatidylcholine).

Negative-ion ESI tandem mass spectrometry of commercially obtained $\mathrm{N}$-monomethyl and $\mathrm{N}, \mathrm{N}$-dimethyl phosphatidylethanolamines derived from egg yolk lecithin demonstrated similar product-ion patterns to those obtained from phosphatidylethanolamines with several lysophospholipidlike anions and carboxylate anion(s). For example, selection and collisional activation of the ion at $\mathrm{m} / \mathrm{z} 731$ (that correspond to $\mathrm{N}$-monomethyl palmitoyl-oleoyl phosphatidylethanolamine) from $N$-monomethyl egg yolk phosphatidylethanolamines yielded two abundant carboxylate anions at $m / z 281$ and 255 (that correspond to oleate and palmitate, respectively) at a peak intensity ratio of greater than 3 (similar intensity ratio of $s n-2$ carboxylate anion versus $s n-1$ carboxylate anion also was observed from examination of 16:0-18:2, 18:0-18:1, and 18:0-18:2 N-monomethyl and $N, N$-dimethyl phosphatidylethanolamines derived from egg yolk lecithin). The peak intensity ratio of carboxylate anions can be used to identify the regiospecificity of asymmetrical $\mathrm{N}$-monomethyl or $\mathrm{N}, \mathrm{N}$-dimethyl phosphatidylethanolamines as described in the preceding text. In addition, four less intense product ions at $\mathrm{m} / \mathrm{z}$ $449,467,475$, and 494 that correspond to $N$-monomethyl lysophosphatidylethanolaminelike anions (spectra not shown) also were present.

In positive-ion ESI mass spectrometry of ethanolamine glycerophospholipids, only the sodiated ion (i.e., $[\mathrm{M}+\mathrm{Na}]^{+}$) is present under the experimental conditions employed. Selection and collisional activation of the sodium adduct that corresponds to sodiated 1-octadecanoyl-2-eicosatetra-5', $8^{\prime}, 11^{\prime}, 14^{\prime}$-enoyl-snglycero-3-phosphoethanolamine (i.e., stearoyl arachidonoyl phosphatidylethanolamine) yielded multiple product ions (Figure 5a) that include $m / z 748$ (that corresponds to the neutral loss of vinyl amine to form sodiated stearoyl arachidonoyl phosphatidic acid), $m / z$ 668 (that corresponds to the neutral loss of the fivemembered amidophosphane), and ions at $m / z 650$ (i.e., $[\mathrm{M}+\mathrm{Na}-141]^{+}$) and 628 (i.e., $[\mathrm{M}+\mathrm{Na}-$ $163]^{+}$) (that correspond to diglyceridelike ions). The $[\mathrm{M}+\mathrm{Na}-141]^{+}$ion peak always is more intense than the $[\mathrm{M}+\mathrm{Na}-163]^{+}$ion peak in ESI-MS/MS of sodiated phosphatidylethanolamine and yet only the

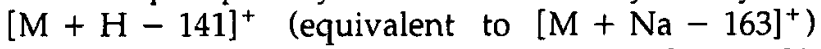
product ion is present from the precursor ion $[\mathrm{M}+\mathrm{H}]^{+}$ in phosphatidylethanolamines that utilize both ESIMS/MS (data not shown) and FAB-MS/MS [21, 23, 25]. The product ions of $m / z 444$ and 464 in ESIMS/MS of sodiated stearoyl arachidonoyl phosphatidylethanolamine likely correspond to the further neutral loss of arachidonic acid and stearic acid, respectively, from the product ion $m / z 748$ (sodiated phosphatidic acid) via formation of a five- or six-membered phosphodiester, respectively (Scheme II). These two product ions provide information about the regiospecificity of $s n-1$ and $s n-2$ constituents of phos- 
Figure 5. Positive-ion ESI tanden mass spectra of sodiated ethanol amine glycerophospholipids. (a) ESIMS/MS of sodiated 1-octadecanoyl2-eicosatetra-5', 8', 11', 14'-enoyl-snglycero-3-phosphoethanolamine. (b) ESI-MS/MS of sodiated 1-O-(Z)octadec - $1^{\prime}$-enyl -2-eicosatetra-5', $8^{\prime}$, $11^{\prime}, 14^{\prime}$-enoyl-sn-glycero-3-phosphoethanolamine (plasmenylethanolamine). Each ethanolamine glycerophospholipid (1 pmol $/ \mu \mathrm{L}$ ) was dissolved in $1: 2$ chloroform-methanol prior to performance of ESI tandem mass spectrometry as described in the Materials and Methods section. a

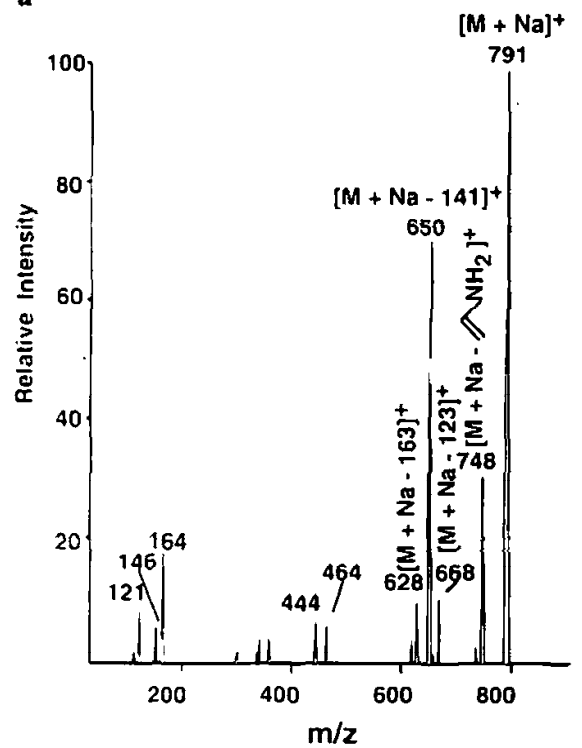

b

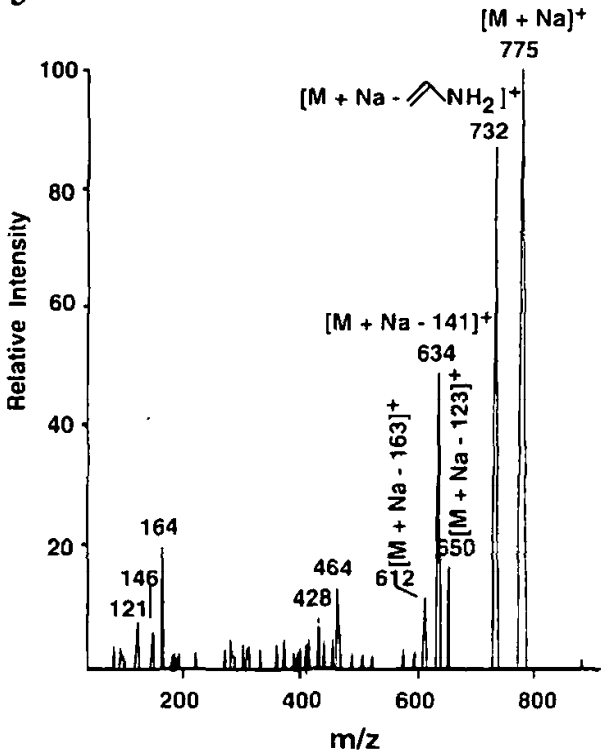

phatidylethanolamine. Because the ratios of these product ions are only slightly different, it is preferable to assign the regiochemistry of the $s n-1$ and $s n-2$ constituent from negative-ion ESI-MS/MS. The ions at $m / z 121,146$, and 164 support the possible pathways proposed in Scheme II. The positive-ion ESI-MS/MS of sodiated plasmenylethanolamine is distinct from that of its sodiated phosphatidylethanolamine counterpart, in the presence of a larger ratio of $m / z 732$ to $m / z 634$ in plasmenylethanolamine (Figure $5 \mathrm{~b}$ ) as compared to the ratio of $m / z \quad 748$ to $m / z \quad 650$ in phosphatidylethanolamine (Figure $5 \mathrm{a}$ ). This difference also has been observed in ESI-MS/MS of other sodiated plasmenylethanolamine and phosphatidylethanolamine molecular species examined such as 16:0-20:4 and 18:1-20:4 ethanolamine glycerophospholipids.

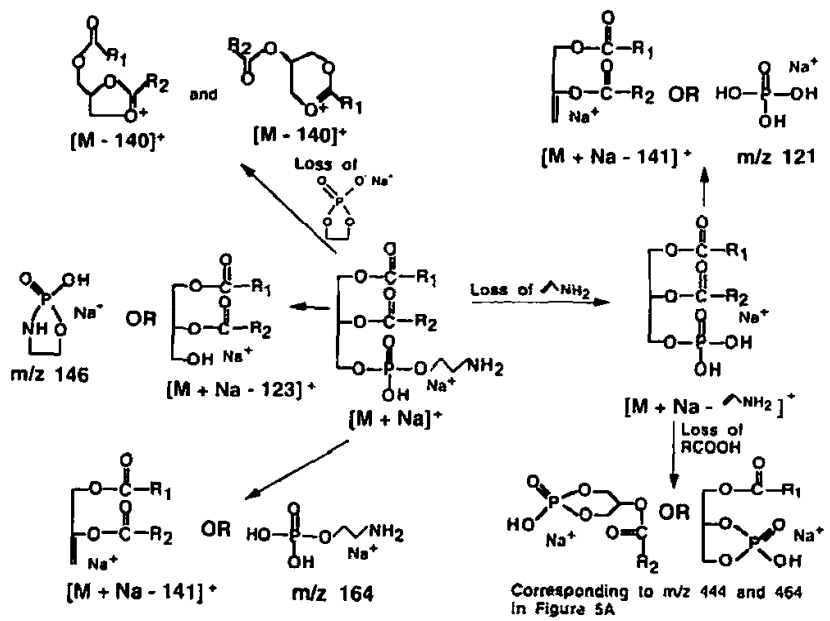

Scheme II. Proposed collision-induced dissociation pathways of sodiated ethanolamine glycerophospholipids after electrospray ionization.

\section{Tandem Mass Spectrometry of Cardiolipin}

Negative-ion ESI mass spectrometry studies of bovine heart cardiolipin demonstrated a major $(\mathrm{m} / \mathrm{z} 724)$ and two minor peaks $(\mathrm{m} / \mathrm{z} 710$ and 737 ) (Figure 6a) that correspond to $[\mathrm{M}-2 \mathrm{H}]^{2-}$ quasimolecular ions of individual cardiolipin molecular species. These results are dramatically different from analyses that employed FAB mass spectrometry, where relatively high energy ionization resulted in the production of the $[\mathrm{M}-\mathrm{H}]^{-}$form of the molecular ion and carboxylate fragment ions [24].

Selection and collisional activation of the [M $2 \mathrm{H}]^{2-}$ ion $(m / z 724)$ after ESI yielded multiple informative product ions (Figure $6 \mathrm{~b}$ ). This product ion pattern differs significantly from that generated from collisional activation of singly charged precursor ions of cardiolipin during FAB ionization [24]. The most abundant product ion, $m / z 279$, is the carboxylate anion of linoleic acid. Because only one product ion peak is observed in the fatty acid region, this result indicates that all four acyl groups of the selected cardiolipin ion $(m / z 724)$ are linoleoyl chains. These results were confirmed by capillary gas chromatography after acid methanolysis of bovine heart cardiolipin, which demonstrated the exclusive presence of linoleic acid in the sample.

Interestingly, after collisional activation, the doubly charged precursor ion $\left([\mathrm{M}-2 \mathrm{H}]^{2-}, m / z 724\right)$ can directly generate two pairs of singly charged product ions (Figure $6 \mathrm{~b}$ and Scheme III). One pair of the product ions $(m / z 279$ and $m / z 1168)$ corresponds to the collision-induced dissociation of the $[\mathrm{M}-2 \mathrm{H}]^{2-}$ ion as shown in Scheme III. The product ion at $m / z 1168$ was further dissociated into product ions, $m / z 888$ and 906 , which correspond to the neutral loss of fatty 
a

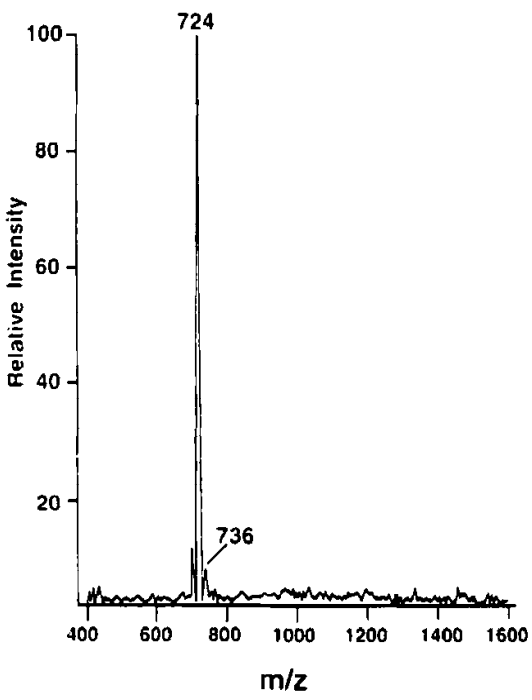

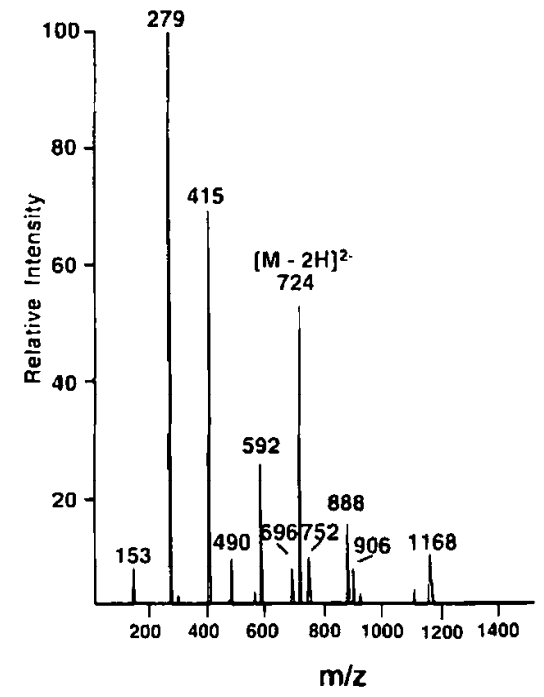

Figure 6. Negative-ion ESI mass spectrometry of cardiolipin. (a) Negative-ion ESI mass spectrum of bovine heart cardiolipins. (b) Negative-ion ESI tandem mass spectrum of selected cardiolipin ion $\left([\mathrm{M}-2 \mathrm{H}]^{2-}, \mathrm{m} / \mathrm{z}\right.$ 724). Bovine heart cardiolipins (1 pmol $/ \mu \mathrm{L}$ ) were dissolved in 1:2 chloroform-methanol prior to performance of ESI mass spectrometry as described in the Materials and Methods section.

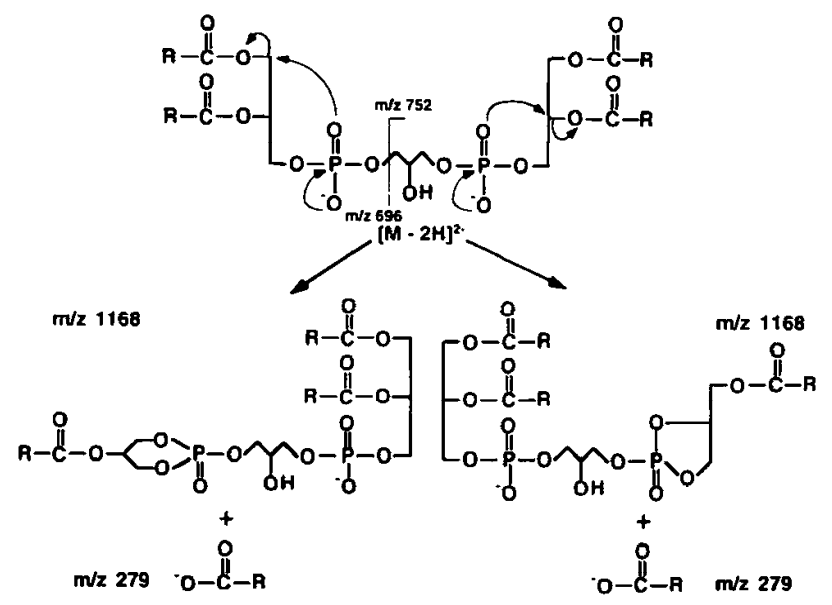

Scheme III. The major collision-induced dissociation pathways of doubly charged cardiolipin anion (i.e., $[\mathrm{M}-2 \mathrm{H}]^{2-}$ ) after electrospray ionization. acid and alkyl ketene, respectively. The other pair of the product ions, $m / z 752$ (that corresponds to the loss of $\mathrm{H}_{2} \mathrm{O}$ from dilinoleoyl phosphatidylglycerol) and $m / z 696$ (that corresponds to dilinoleoyl phosphate), are generated from collision-induced dissociation of the adjacent phosphodiester linkage (Scheme III). The dilinoleoyl phosphatidylglycerol analog (i.e., $m / z$ 752), generated from precursor ion $\left([\mathrm{M}-2 \mathrm{H}]^{2-}, m / z 724\right)$ was further dissociated into an ion $(m / z 490)$ that corresponds to the neutral loss of alkyl ketene, whereas the dilinoleoyl phosphate $(m / z$ 696) was dissociated further into the lysophosphatidic acid analog $(\mathrm{m} / \mathrm{z}$ $415)$ through the neutral loss of fatty acid. The product ion at $m / z 592$ resulted from dissociation of the precursor ion $\left([\mathrm{M}-2 \mathrm{H}]^{2-}\right)$ through the neutral loss of alkyl ketene (i.e., $\left[\mathrm{M}-2 \mathrm{H}-\mathrm{R}^{\prime} \mathrm{C}=\mathrm{C}=\mathrm{O}\right]^{2-}$ ). The product ion at $m / z 153$ corresponds to the loss of $\mathrm{H}_{2} \mathrm{O}$ from glycerol phosphate.

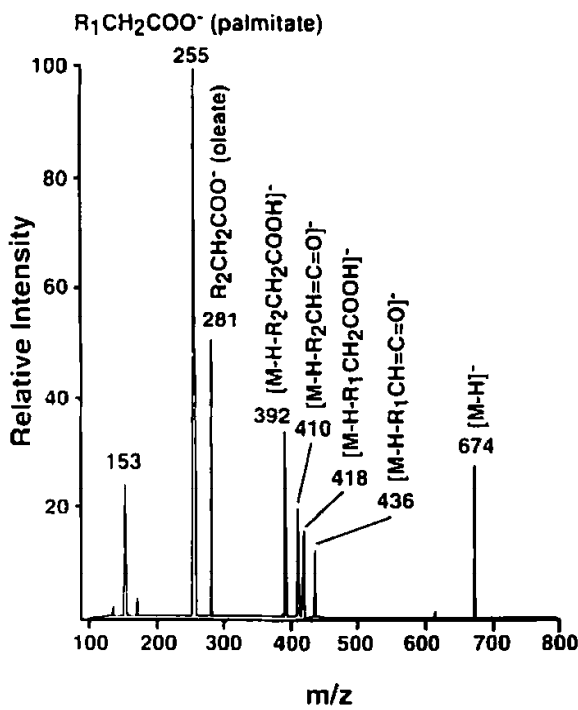

b

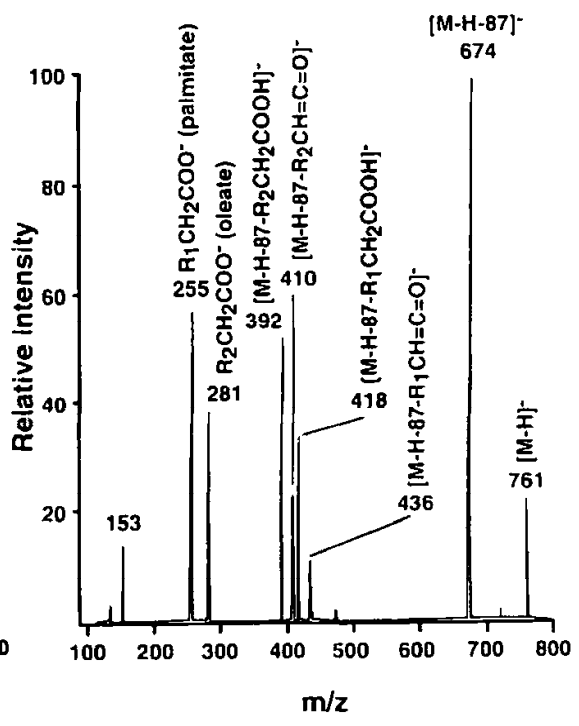

Figure 7. Negative-ion ESI tandem mass spectra of deprotonated phosphatidic acid and phosplatidylserine. (a) ESI-MS/MS of 1-hexadecanoyl-2-octadec-9'-enoyl-silglycero-3-phosphate. (b) ESI-MS/MS of deprotonated 1-hexadecanoyl-2-octadec9'-enoyl-sn-glycero-3-phosphoserine. Each phospholipid $(0.2 \mathrm{pmol} / \mu \mathrm{L})$ was dissolved in 1:2 chloroform-methanol prior to acquisition of the ESI-MS/MS spectra as described in the Materials and Methods section. 


\section{Tandem Mass Spectrometry of} Anionic Phospholipids

The negative-ion ESI-MS/MS spectra of other anionic phospholipids including phosphatidylserine, phosphatidylinositol, phosphatidylglycerol, and phosphatidic acid are nearly identical to those obtained via FAB-MS/MS [16, 21, 24], but can be obtained with a much greater sensitivity via ESI-MS/MS. As examples, the negative-ion ESI-MS/MS spectra of phosphatidic acid and phosphatidylserine from 1 pmol of material (consumed) are shown in Figure 7.

\section{Conclusion}

The results presented in this study demonstrate the utility of tandem mass spectrometry of volatilized ions after electrospray ionization for the structural determination of phospholipids. ESI product-ion spectra can be obtained readily by utilizing $\leq 5 \mathrm{pmol}$ of either synthetic or biologically derived phospholipids with a mass-selected window of less than 2 mass units. Although many of the regioisomeric assignments based on the relative peak intensity ratios of $s n-1$ and $s n-2$ carboxylate ions were similar to those observed by using FAB tandem mass spectrometry, collisioninduced dissociation of precursor ions derived from electrospray ionization often resulted in novel production patterns. Collectively, these results underscore the unrecognized potential of electrospray ionization tandem mass spectrometry for the structural determination of individual molecular species of phospholipids in biologic samples from picomole amounts of material.

\section{Acknowledgment}

This research was supported by NIH grant HL41250 and the Washington University Mass Spectrometry Resource NIH Grant RR95418.

\section{References}

1. Han, X.; Gross, R. W. Proc. Natl. Acad. Sci. USA 1994, 91, 10635-10639.

2. Weintraub, S. T.; Pinckard, R. N.; Hail, M. Rapid Conmmun. Mass Spectrom. 1991, 5, 309-311.

3. Kerwin, J. L.; Tuininga, A.; Ericsson, L. H. /. Lipid Res. 1994, 35, 1102-1114.

4. Kim, H.-Y.; Wang, T.-C. L.; Ma, Y.-C. Anal. Chem. 1994, 66 , 3977-3982.

5. Cole, M. J.; Enke, C. G. Anal. Chem. 1991, 63, 1032-1038.

6. Kayganich, K. A.; Murphy, R. C. Anal. Chem. 1992, 64, 2965-2971.

7. Bryant, D. K.; Orlando, R. C.; Fenselau, C. Anal. Chem. 1991, $63,1110-1114$.

8. Jensen, N. J.; Gross, M. L. Mass Spectrom. Rev. 1988, 7, 41-69.

9. Benfenati, E.; Reginato, R. Biomed. Mass Spectrom. 1985, 12 , 643-651.

10. Ayanoglu, E.; Wegmann, A.; Pilet, O.; Marbury, G. D.; Hass, J. R.; Djerassi, C. I. Am. Chem. Soc, 1984, 106, 5246-5251.

11. Matsubara, T.; Hayashi, A. Prog. Lipid Res. 1991, 30, 301-322.

12. Patton, G. M.; Fasulo, J. M.; Robins, S. J. J. Lipid Res. 1982, 23. 190-196.

13. Gross, R. W. Biochemistry 1984, 23, 158-165.

14. Han, X.; Zupan, L. A.; Hazen, S. L.; Gross, R. W. Anal. Biochem. 1992, 200, 119-124.

15. Fink, K. L.; Gross, R. W. Circ. Res. 1984, 55, 585-594.

16. Jensen, N. J.; Tomer, K. B.; Gross, M. L. Lipids 1986, 21, 580-588.

17. Münster, H.; Budzikiewicz, H. Biol. Chem. Hoppe-Seyler 1988, 369, 303-308.

18. Kayganich, K.; Murphy, R. C. I. Am. Soc. Mass Spectrom. 1991, $2,45-54$.

19. Zirrolli, J. A.; Clay, K. L.; Murphy, R. C. Lipids 1991, 26, 1112-1116.

20. Huang, Z.-H.; Gage, D. A.; Sweeley, C. C. J. Am. Soc. Mass Spectrom. 1992, 3, 71-78.

21. Murphy, R. C. Mass Spectrometry of Lipid; Plenum Press: New York, 1993; pp 290.

22. Kim, H.-Y.; Salem, N., Jr. Anal. Clem, 1986, 58, 9-14.

23. Haroldsen, P. E.; Gaskell, S. J. Biomed. Environ. Mass Spectrom. $1989,18,439-444$.

24. Jensen, N. J.; Tomer, K. B.; Gross, M. L. Lipids 1987, 22 , 480-489.

25. Heller, D. N.; Murphy, C. M.; Cotter, R. J.; Fenselau, C.; Uy, O. M. Anal. Chem. 1988, 60, 2787-2791. 